\title{
The Correlation Between Esophageal and Abdominal Pressures in Mechanically Ventilated Patients Undergoing Laparoscopic Surgery
}

\author{
Anees Sindi MBChB, Tom Piraino RRT, Waleed Alhazzani MD, Marcel Tunks MD, \\ Mazen Faden MBChB, Jinhui Ma MSc, Lehana Thabane PhD, and Maureen Meade MD
}

\begin{abstract}
BACKGROUND: Intrathoracic pressure in patients undergoing laparoscopic surgery may be affected by intra-abdominal pressure during surgery. We investigated the relationship between intraabdominal pressure $\left(\mathbf{P}_{\mathrm{abd}}\right)$ and esophageal pressure $\left(\mathbf{P}_{\mathrm{es}}\right)$ in mechanically ventilated patients undergoing laparoscopic surgery. METHODS: We prospectively studied 43 consenting patients over $18 \mathrm{y}$ of age who were scheduled for elective laparoscopic surgery with plans for intra-operative intubation and paralysis. After establishing a good level of inter-observer agreement on $\mathbf{P}_{\mathrm{es}}$ measurements, $P_{e s}$ was measured by one observer for each patient using an esophageal catheter. $P_{a b d}$ and $P_{e s}$ were recorded before and after abdominal insufflation. We used regression analysis to model the relationship between $P_{a b d}$ and $P_{e s}$. RESULTS: Patients' ages varied from 22 to $78 \mathrm{y}$, with a mean of $53.2 \pm 14.6 \mathrm{y}$. Body mass index (BMI) varied from 13.7 to $60.5 \mathrm{~kg} / \mathrm{m}^{2}$, with a mean of 33.7 \pm 10.5. PEEP was $5-7 \mathrm{~cm} \mathrm{H}_{2} \mathrm{O}$ for 19 patients and $0 \mathrm{~cm} \mathrm{H}_{2} \mathrm{O}$ for the remainder. Most patients underwent gastric bypass surgery $(n=11)$; others underwent hernia repair $(n=9)$, colon resection $(n=7)$, cholecystectomy $(n=6)$, and various other surgeries $(n=10)$. Using univariate analyses, baseline $P_{\text {abd }}$ was significantly correlated with baseline $P_{\text {es }}$ (estimate of model coefficient $[95 \% \mathrm{CI}]$ : $\left.0.79[0.36-1.21], \mathrm{R}^{2}=0.24, P=.001\right)$, as was BMI $\left(0.29[0.19-0.40], \mathrm{R}^{2}=0.41, P<.001\right)$. However, a multivariable analysis showed no significant correlation with baseline $P_{\text {abd }}(0.10$ [ -0.46 to 0.65 ], $P=.73)$, whereas BMI remained highly significant $(0.27[0.11-00.43], P=.001)$ with $R^{2}=0.40$. Due to unexpected uniformity of abdominal inflation pressures (generally $20.4 \mathrm{~cm} \mathrm{H}_{2} \mathrm{O}$ ) during surgery, data were not amenable to assessment of correlation between changes in abdominal and esophageal pressures after inflation. CONCLUSION: There was a limited correlation between baseline $P_{e s}$ and $P_{a b d}$ in patients undergoing elective laparoscopic surgery, suggesting a limited value of $\mathbf{P}_{\text {abd }}$ measurements in the management of mechanically ventilated patients. Key words: esophageal pressure; abdominal pressure; transpulmonary pressure; abdominal insufflation; prospective study. [Respir Care 2014;59(4):491-496. (C) 2014 Daedalus Enterprises]
\end{abstract}

\section{Introduction}

Transpulmonary pressure during positive-pressure ventilation refers to the summation of positive forces that

\footnotetext{
Drs Sindi and Faden are affiliated with the Department of Anesthesia and Critical Care, King Abdulaziz University, Jeddah, Saudi Arabia. Mr Piraino and Drs Alhazzani and Meade are affiliated with the Division of Critical Care, Department of Anesthesia; Dr Tunks is affiliated with the Division of Respirology, Department of Medicine; and Ms Ma and Drs Thabane and Meade are affiliated with the Department of Clinical Epidemiology and Biostatistics, McMaster University, Hamilton, Ontario, Canada. Ms Ma and Dr Thabane are also affiliated the Biostatistics Unit, St. Joseph's Healthcare Hamilton, Hamilton, Ontario, Canada.
}

inflate the lungs (airway inflation pressure) and negative forces that prevent the lungs from collapsing (pleural pres-

\footnotetext{
This research was supported by the Father Sean O'Sullivan Research Centre and the Firestone Institute for Respiratory Health, St. Joseph's Healthcare Hamilton. The authors have disclosed no conflicts of interest.

Dr Sindi and Mr Piraino are co-first authors.

Correspondence: Anees Sindi MBChB, Department of Anesthesia and Critical Care, King Abdulaziz University, Jeddah 22254, Saudi Arabia. E-mail: ansindi@me.com.
}

DOI: $10.4187 /$ respcare. 02287 
sure). In healthy subjects with normal lungs, transpulmonary pressure is slightly positive, which serves to maintain a functional residual capacity. For critically ill patients with ARDS, maintaining an "open lung" is important to minimize end-expiratory atelectasis, which might otherwise contribute to ventilator-induced lung injury and possibly the risk of death. ${ }^{1-5}$ Esophageal pressure manometry is a method to estimate pleural pressure and thus derive the transpulmonary pressure..$^{3,6-9}$

Investigations of a role for esophageal manometry in guiding mechanical ventilation show promise. The application of PEEP to the airways, as guided by the measurement of esophageal pressure to estimate transpulmonary pressure, has demonstrated improved respiratory system compliance and improved oxygenation in critically ill adults with ARDS.5,6 Despite these reported successes, clinical uptake has been limited. This may be related, in part, to lack of familiarity with the technology and the invasive nature of esophageal probe insertion. ${ }^{3-10}$ The availability of accurate and familiar surrogate measurements for esophageal pressure could, in theory, help to overcome these deterrents. One potential surrogate is abdominal pressure $\left(\mathrm{P}_{\mathrm{abd}}\right)$, which can be measured at the bedside as bladder pressure. However, a previous study has shown a significant but limited correlation between gastric pressure $\left(\mathrm{P}_{\mathrm{ga}}\right)$ and esophageal pressure $\left(\mathrm{P}_{\mathrm{es}}\right)$ in patients with acute respiratory failure $\left(\mathrm{R}^{2}=0.354, P<.0001\right){ }^{3}$

The main objective of this study was to explore the correlation between $\mathrm{P}_{\text {abd }}$ (as measured via an abdominal insufflator) and $\mathrm{P}_{\mathrm{es}}$ (as measured via esophageal pressure manometry) in surgical patients undergoing laparoscopic surgery. Establishing a correlation between $\mathrm{P}_{\mathrm{abd}}$ and $\mathrm{P}_{\mathrm{es}}$ may stimulate researchers to look at the use of bedside abdominal pressure measurements (bladder pressure) rather than esophageal pressure to optimize the application of PEEP in the ICU.

\section{Methods}

\section{Study Design}

We conducted a prospective single-cohort observational study at St. Joseph's Healthcare Hamilton (SJHH) in Ontario, Canada. The study underwent full review and received approval by the SJHH Research Ethics Board.

\section{Patients}

Patient recruitment began in January 2011 and stopped at the end of March 2011. We screened patients in the adult pre-operative clinic for the following inclusion criteria: scheduled elective laparoscopic surgery, age $>18 \mathrm{y}$, and a plan for intra-operative intubation and paralysis. We excluded patients with any contraindication to esophageal

\section{QUICK LOOK}

\section{Current knowledge}

Esophageal manometry estimates pleural pressure and can be used to derive transpulmonary pressure. Abdominal pressure as determined by bladder pressure has also been used to determine transpulmonary pressure. Laparoscopic abdominal surgery uses carbon dioxide insufflation to optimize the surgical field, increasing intraabdominal pressure.

\section{What this paper contributes to our knowledge}

In a group of patients without respiratory disease undergoing laparoscopic surgery, there was a significant but limited relationship between esophageal and abdominal pressures. Intra-abdominal pressure cannot predict esophageal pressure but can provide complementary information useful in setting mechanical ventilation.

manometry, including esophageal rupture, esophageal varices, esophagectomy, or recent esophageal or gastric surgery. Measurements in the operating room were obtained in parallel with patients' recruitment in the pre-operative clinic during these 3 months depending on the surgery time and date.

\section{Measurements}

In the operating room, study patients were sedated, paralyzed, and mechanically ventilated, in accordance with both routine surgical care and the study protocol. All patients were positioned supine. We reviewed 3 distinct protocols for esophageal catheter insertion ${ }^{3,5,7-9}$ and chose the technique used recently by Talmor et $\mathrm{al}^{3,5}$ in their management of critically ill adults with ARDS. A study investigator inserted the esophageal catheter (adult esophageal balloon catheter set, CooperSurgical, Trumbull, Connecticut). The catheter was inserted to $60 \mathrm{~cm}$ and connected to a pressure transducer, and pressure waveforms and measurements were displayed on an associated monitor. Placement in the stomach is usually confirmed in passively breathing patients by the absence of cardiac oscillations, a rise in pressure during a positive-pressure breath delivered by the ventilator, and a transient increase in pressure during a gentle compression of the abdomen, and there should be no resistance (due to coiling) when removing the catheter stylet. To avoid contaminating the surgical field, gentle compression of the abdomen was omitted. The catheter was then withdrawn to $40 \mathrm{~cm}$ and assumed to be placed in the lower third of the esophagus only if cardiac oscillations were noted. Two investigators (AS and TP) obtained 
$\mathrm{P}_{\mathrm{es}}$ measurements independently for the first 5 patients to assess inter-rate agreement. Inter-rate agreement was 0.44 (moderate agreement) using Cohen's kappa statistics method, ${ }^{11}$ and the investigators further unified their procedures accordingly. A study by Pelosi et al ${ }^{12}$ showed that pleural pressure could be estimated by measuring esophageal pressure directly at mid-lung height, which we did in our study. However, their study was conducted in animal models. In humans, the effect of mediastinal weight on $\mathrm{P}_{\mathrm{es}}$ measured in healthy volunteers in the supine position was $\sim 3 \mathrm{~cm} \mathrm{H}_{2} \mathrm{O}^{13}$ For this reason, we subtracted $3 \mathrm{~cm} \mathrm{H}_{2} \mathrm{O}$ from our measured $\mathrm{P}_{\mathrm{es}}$ values.

After the attending surgeon inserted an abdominal insufflator (PneumoSure 45L insufflator, Stryker, Kalamazoo, Michigan), a study investigator recorded the baseline esophageal pressure at end of expiration, as identified from the waveform display. Next, the surgeon insufflated the abdomen to achieve adequate visualization in accordance with routine surgical management, and a post-insufflation esophageal pressure measurement was recorded, again at end of expiration. Foley catheter insertion was uncommon; therefore, abdominal pressures were directly recorded for all patients from the insufflator screen by a study investigator.

\section{Data Collection and Statistical Analysis}

Study data for each patient include demographics, primary diagnosis, comorbidities, and pressure data before and after abdominal insufflation. Data were entered into a password-protected database.

The sample size for this study was determined based upon the following statistical considerations. We needed a sample size that would allow us to fit a regression with 3 predictor variables of interest: body mass index (BMI), PEEP, and baseline pressures. Our original plan was to study 50 patients over 4-6 months; however, with delays related to equipment availability and cancelled surgeries, we terminated the study after including 43 patients over 3 months.

Baseline and study data are summarized using descriptive summary measures, expressed as mean \pm SD for continuous variables and count frequencies (percentages) for categorical variables. We conducted univariate and multiple linear regression analyses using insufflation $\mathrm{P}_{\mathrm{es}}$ measurement as the outcome with baseline $\mathrm{P}_{\mathrm{es}}, \mathrm{P}_{\mathrm{abd}}$, and BMI as independent variables. We were unable to investigate the correlation between changes in $\mathrm{P}_{\mathrm{es}}$ with change in $\mathrm{P}_{\mathrm{abd}}$ due to an unexpected lack of variability in insufflation pressures. Specifically, in contrast to prior experience, surgeons routinely inflated the abdomen to $\sim 15 \mathrm{~mm} \mathrm{Hg}$ (20.4 $\mathrm{cm} \mathrm{H}_{2} \mathrm{O}$ ). We assessed for collinearity (correlations among predictors) using a scatter plot and variance inflation factor (VIF), and considered variables with VIF $>10$

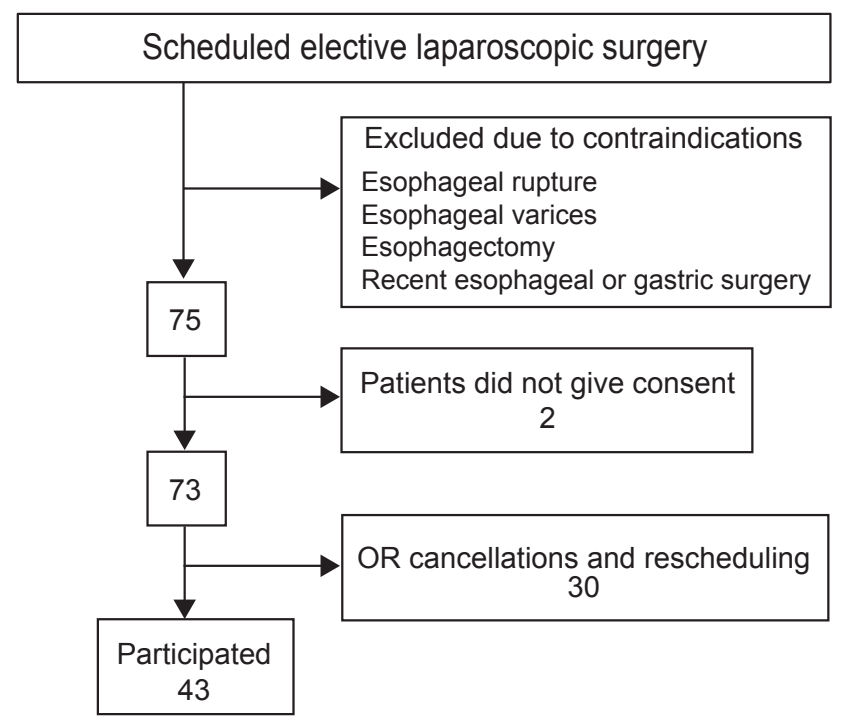

Fig. 1. Trial enrollment. $\mathrm{OR}=$ operating room.

as collinear. ${ }^{14}$ Model assumptions and goodness of fit were evaluated using the residual plot and $\mathrm{R}^{2}$, respectively. Results from the regression analysis are reported as an estimate of the coefficient and the corresponding 95\% CI values and associated $P$ values. All analyses were performed using statistics software (Stata 10, StataCorp, College Station, Texas).

\section{Results}

Seventy-three patients consented to participate in the trial, and 43 were included (Fig. 1). Thirty patients were not included as a result of surgical cancellations, with or without rescheduling within the allotted time frame of the study. Tables 1 and 2 summarize baseline patient characteristics. Patients' ages varied from 22 to $78 \mathrm{y}$, with a mean of $53.2 \pm 14.6 \mathrm{y}$. BMI varied from 13.7 to 60.5 $\mathrm{kg} / \mathrm{m}^{2}$, with a mean of $33.7 \pm 10.5 \mathrm{~kg} / \mathrm{m}^{2}$. Fifty-one percent of the patients were classified as obese. All patients were ventilated using either pressure or volume control mode. Nineteen patients were on PEEP during surgery; levels varied between 5 and $7 \mathrm{~cm} \mathrm{H}_{2} \mathrm{O}$. Most patients underwent gastric bypass surgery (11/43). Mean baseline $\mathrm{P}_{\mathrm{es}}$ and $\mathrm{P}_{\mathrm{abd}}$ were $9.5 \pm 4.7$ and $7.8 \pm 3.0 \mathrm{~cm} \mathrm{H}_{2} \mathrm{O}$, respectively.

Using univariate analyses, we found that baseline $\mathrm{P}_{\mathrm{abd}}$ is significantly correlated with baseline $\mathrm{P}_{\mathrm{es}}$ (estimate of model coefficient [95\% CI]: $0.79[0.36-1.21], \mathrm{R}^{2}=0.24$, $P=.001)$, as was BMI $\left(0.29[0.19-0.40], \mathrm{R}^{2}=0.41\right.$, $P<.001$ ) (Fig. 2). However, a multivariable analysis showed no significant correlation with baseline $\mathrm{P}_{\mathrm{abd}}(0.10$ [ -0.46 to 0.65$], P=.731)$, whereas BMI remained highly significant $(0.27$ [0.11-0.43], $P=.001)$ with $\mathrm{R}^{2}=0.40$ (Table 3). However, the correlation between post-insuffla- 
Table 1. Baseline Clinical Statistics

\begin{tabular}{lc}
\hline \hline \multicolumn{1}{c}{ Variable } & Summary Statistics \\
\hline Age, mean \pm SD y & $53.2 \pm 14.6$ \\
BMI, mean \pm SD kg/m² & $33.7 \pm 10.5$ \\
Intervention, $n(\%)$ & \\
Colonic resection & $7(16.3)$ \\
Gastric bypass & $11(25.6)$ \\
Hernia repair & $9(20.9)$ \\
Hysterectomy & $3(7)$ \\
Cholecystectomy & $6(14)$ \\
Myotomy & $1(2.3)$ \\
Nephrectomy & $3(7)$ \\
Prostate resection & $2(4.7)$ \\
Salpingo-oophorectomy & $1(2.3)$ \\
\hline$(n=43)$ & \\
BMI $=$ body mass index & \\
\hline
\end{tabular}

Table 2. Ventilatory Settings

\begin{tabular}{lc}
\hline \hline \multicolumn{1}{c}{ Variable } & Summary Statistics \\
\hline Ventilatory mode, $n(\%)$ & \\
$\quad$ Pressure control & $4(9.3)$ \\
$\quad$ Volume control & $39(90.7)$ \\
$\mathrm{f}$, mean $\pm \mathrm{SD}$ & $10.5 \pm 1.5$ \\
$\mathrm{PEEP}$, mean $\pm \mathrm{SD}$ & $2.7 \pm 2.8$ \\
$\mathrm{~F}_{\mathrm{IO}}$, mean $\pm \mathrm{SD}$ & $0.5 \pm 0.1$ \\
$\mathrm{P}_{\text {abd }}$, mean $\pm \mathrm{SD}$ cm $\mathrm{H}_{2} \mathrm{O}$ & \\
$\quad$ Baseline & $7.8 \pm 3$ \\
$\quad$ Post-insufflation & $20.9 \pm 1.2$ \\
$\quad \Delta \mathrm{P}_{\text {abd }}$ & $13.1 \pm 3$ \\
$\mathrm{P}_{\mathrm{es}}$, mean $\pm \mathrm{SD}$ cm $\mathrm{H}_{2} \mathrm{O}$ & \\
$\quad$ Baseline & $9.5 \pm 4.7$ \\
$\quad$ Post-insufflation & $11.3 \pm 6.2$ \\
$\Delta \mathrm{P}_{\mathrm{es}}$ & $1.8 \pm 3.6$ \\
& \\
\hline$(n=43)$ & \\
$\mathrm{f}=$ breathing frequency (breaths/min $)$ & \\
$\mathrm{P}_{\text {abd }}=$ intra-abdominal pressure & \\
$\Delta \mathrm{P}_{\text {abd }}=$ difference between the post-insufflation and baseline $\mathrm{P}_{\text {abd }}$ measurements \\
$\mathrm{P}_{\mathrm{es}}=$ esophageal pressure \\
$\Delta \mathrm{P}_{\mathrm{es}}=$ difference between the post-insufflation and baseline $\mathrm{P}_{\mathrm{es}}$ measurements \\
\hline
\end{tabular}

tion pressures and changes in abdominal and esophageal pressures could not be calculated as a result of an insufficient range of values in post-insufflation abdominal pressures and change in abdominal pressures.

\section{Discussion}

In this prospective observational study, we found a significant but limited correlation between baseline $\mathrm{P}_{\mathrm{es}}$ and $\mathrm{P}_{\mathrm{abd}}$ in patients undergoing laparoscopic surgery (see Table 3). This correlation was not statistically significant after adjusting for BMI.
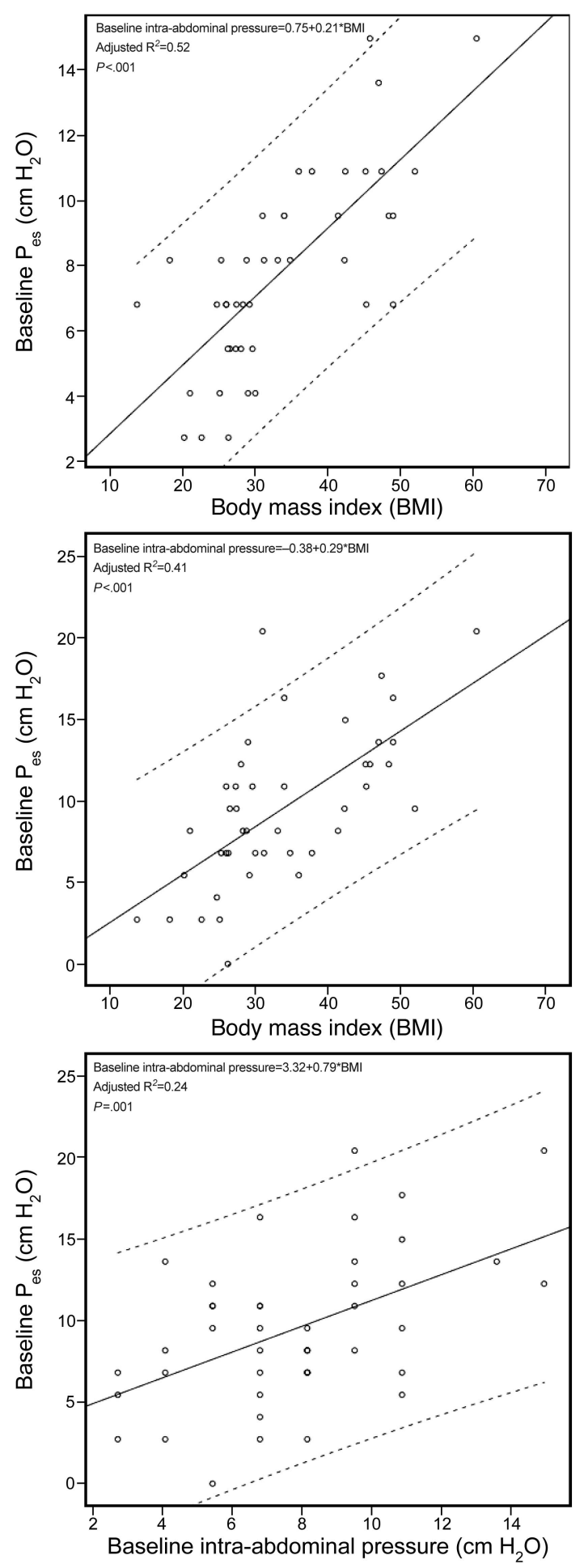

Fig. 2. Esophageal and intra-abdominal pressures at baseline related to body mass index (BMI). $\mathrm{P}_{\mathrm{es}}=$ esophageal pressure.

To our knowledge, there are no studies that have previously addressed this question in the same population. However, a relevant study ${ }^{3}$ reported a significant correlation between $\mathrm{P}_{\mathrm{ga}}$ as an indirect method for measuring in- 
Table 3. Linear Regression With Internal Model Validation

\begin{tabular}{|c|c|c|c|c|}
\hline \multirow[b]{2}{*}{ Outcome } & \multirow[b]{2}{*}{ Covariate } & \multicolumn{3}{|c|}{ Multiple Regression } \\
\hline & & $\begin{array}{l}\text { Coefficient } \\
\text { (95\% CI) }\end{array}$ & $P$ & $\begin{array}{c}\text { Adjusted } \\
\mathrm{R}^{2}\end{array}$ \\
\hline \multirow{7}{*}{ 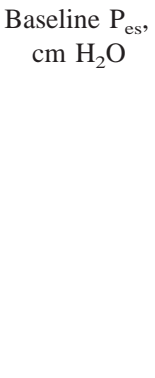 } & Intercept & $3.32(-0.23$ to 6.88$)$ & .066 & 0.24 \\
\hline & $\begin{array}{l}\text { Baseline } \mathrm{P}_{\mathrm{abd}} \\
\quad \mathrm{cm} \mathrm{\textrm {H } _ { 2 } \mathrm { O }}\end{array}$ & $0.79(0.36-1.21)$ & .001 & \\
\hline & Intercept & $-0.38(-4.16$ to 3.40$)$ & .840 & 0.41 \\
\hline & BMI & $0.29(0.19-0.40)$ & $<.001$ & \\
\hline & Intercept & $-0.45(-4.29$ to 3.39$)$ & .814 & 0.40 \\
\hline & BMI & $0.27(0.11-0.43)$ & .001 & \\
\hline & $\begin{array}{l}\text { Baseline } \mathrm{P}_{\text {abd }} \\
\mathrm{cm} \mathrm{H}_{2} \mathrm{O}\end{array}$ & $0.10(-0.46$ to 0.65$)$ & .731 & \\
\hline
\end{tabular}

Baseline $\mathrm{P}_{\mathrm{es}}=$ esophageal pressure at baseline

Baseline $\mathrm{P}_{\mathrm{abd}}=$ intra-abdominal pressure at baseline

$\mathrm{BMI}=$ body mass index

tra-abdominal pressure and $\mathrm{P}_{\mathrm{es}}$ in patients with acute respiratory failure in the ICU $\left(\mathrm{R}^{2}=0.354, P<.0001\right)$. Their results were similar to ours even though their re-

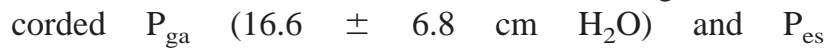
(17.5 $\pm 5.7 \mathrm{~cm} \mathrm{H}_{2} \mathrm{O}$ ) were different from our recorded $\mathrm{P}_{\mathrm{abd}}\left(7.8 \pm 3 \mathrm{~cm} \mathrm{H}_{2} \mathrm{O}\right)$ and $\mathrm{P}_{\mathrm{es}}\left(9.5 \pm 4.7 \mathrm{~cm} \mathrm{H}_{2} \mathrm{O}\right)$. The $\mathrm{P}_{\mathrm{es}}$ measurements in their study were done at the end of exhalation, as was done in our study. However, their mean PEEP level was $14.2 \mathrm{~cm} \mathrm{H}_{2} \mathrm{O}$, and ours was only $2.7 \mathrm{~cm} \mathrm{H} \mathrm{H}_{2} \mathrm{O}$. It is uncertain whether the $\mathrm{P}_{\mathrm{es}}$ levels recorded in their study are a result of the application of airway pressure or are due to secondary causes related to lung injury. In the same study, however, $\mathrm{P}_{\mathrm{es}}$ was not correlated with obesity as assessed by BMI at end of expiration $\left(\mathrm{R}^{2}=0.051, P=.069\right)$, which is in contrast to what we found (95\% CI 0.29 [0.19-0.40], $\left.\mathrm{R}^{2}=0.41, P<.001\right)$. This is likely due to the significant difference between our recorded $\mathrm{P}_{\mathrm{es}}\left(9.5 \pm 4.7 \mathrm{~cm} \mathrm{H}_{2} \mathrm{O}\right)$ and their recorded $\mathrm{P}_{\mathrm{es}}$ (17.5 $\pm 5.7 \mathrm{~cm} \mathrm{H}_{2} \mathrm{O}$ ), which raises the possibility that this correlation between $\mathrm{P}_{\mathrm{es}}$ and $\mathrm{BMI}$ may not exist at higher levels of $\mathrm{P}_{\mathrm{es}}$.

Does this mean that intra-abdominal pressure measurements have a minimal role in the assessment of mechanically ventilated patients? It has previously been shown that intra-abdominal pressures $>12 \mathrm{~cm} \mathrm{H}_{2} \mathrm{O}$ increase chestwall elastance in two thirds of patients. ${ }^{15}$ However, chestwall elastance does not correlate well with $\mathrm{P}_{\mathrm{es}}$ at end of expiration $\left(\mathrm{R}^{2}=0.011, P=.05\right) .{ }^{3}$ Therefore, although $\mathrm{P}_{\mathrm{abd}}$ may not be valuable in predicting $\mathrm{P}_{\mathrm{es}}$, it may still provide complementary information that may be useful in the management of patients receiving mechanical ventilation.

Favorable conditions were present in this study to investigate these relationships: Patient positioning and measurement techniques were consistent, $\mathrm{P}_{\mathrm{abd}}$ was measured directly rather than estimated through gastric or bladder measurements, and all subjects received neuromuscular blockade to eliminate the effect of diaphragmatic tone. However, our study has some noteworthy limitations. We had hoped to include correlation data after abdominal insufflation. Unfortunately, post-insufflation $\mathrm{P}_{\mathrm{abd}}$ measurements were far more uniform than anticipated. The lack of variability in post-insufflation $\mathrm{P}_{\mathrm{abd}}$ pressures made the correlation between post-insufflation $\mathrm{P}_{\mathrm{es}}$ and $\mathrm{P}_{\mathrm{abd}}$ or the changes in $\mathrm{P}_{\mathrm{es}}$ and $\mathrm{P}_{\mathrm{abd}}$ statistically challenging and unreliable. The generalizability of our findings to other settings is also limited. Although there was a difference in the ventilation modes used (pressure or volume), the mode would not impact baseline end-expiratory values of $\mathrm{P}_{\mathrm{es}}$. However, some patients had low levels of PEEP applied and others did not, and this may have affected baseline levels of $\mathrm{P}_{\mathrm{es}}$. Unfortunately, plateau airway pressures were not documented because we were interested in end-expiratory values. If we had measured inspiratory plateau airway and esophageal pressures, we could have included much more valuable data related to lung and chest-wall mechanics.

In a recent landmark ARDS trial, 5 esophageal balloon manometry was used to select PEEP levels to minimize end-expiratory atelectasis. It was hypothesized that maintaining a positive end-expiratory transpulmonary pressure would result in better lung recruitment and less atelectrauma. This novel approach resulted in greater PEEP levels than reported for the control strategy ${ }^{16}$ and better oxygenation, and it appeared to improve survival, although the mortality analyses were limited by a very small number of deaths. ${ }^{17}$ The goal of determining a correlation between $\mathrm{P}_{\mathrm{abd}}$ and $\mathrm{P}_{\mathrm{es}}$ is to provide a surrogate method of estimating transpulmonary pressure.

\section{Conclusions}

In conclusion, we found a significant but limited correlation between baseline $\mathrm{P}_{\mathrm{es}}$ and $\mathrm{P}_{\mathrm{abd}}$ in patients undergoing laparoscopic surgery. Further investigations to elucidate the relationships between abdominal and pleural pressure measurements in critical illness may serve to refine mechanical ventilation protocols.

\section{ACKNOWLEDGMENTS}

We thank Toni Tidy and Dr Marc de Somer for their kind contribution to this project.

\section{REFERENCES}

1. Briel M, Meade M, Mercat A, Brower RG, Talmor D, Walter SD, et al. Higher vs lower positive end-expiratory pressure in patients with acute lung injury and acute respiratory distress syndrome: systematic review and meta-analysis. JAMA 2010;303(9):865-873. 


\section{Correlation Between $\mathrm{P}_{\text {es }}$ And $\mathrm{P}_{\text {abd }}$ In Mechanically Ventilated Patients}

2. Muscedere JG, Mullen JB, Gan K, Slutsky AS. Tidal ventilation at low airway pressures can augment lung injury. Am J Respir Crit Care Med 1994;149(5):1327-1334.

3. Talmor D, Sarge T, O’Donnell CR, Ritz R, Malhotra A, Lisbon A, Loring SH. Esophageal and transpulmonary pressures in acute respiratory failure. Crit Care Med 2006;34(5):1389-1394.

4. Sarge T, Talmor D. Transpulmonary pressure: its role in preventing ventilator-induced lung injury. Minerva Anestesiol 2008;74(6):335-339.

5. Talmor D, Sarge T, Malhotra A, O'Donnell CR, Ritz R, Lisbon A, et al. Mechanical ventilation guided by esophageal pressure in acute lung injury. N Engl J Med 2008;359(20):2095-2104.

6. Piraino T, Cook DJ. Optimal PEEP guided by esophageal balloon manometry. Respir Care 2011;56(4):510-513.

7. Baydur A, Sassoon CS, Carlson M. Measurement of lung mechanics at different lung volumes and esophageal levels in normal subjects: effect of posture change. Lung 1996;174(3):139-151.

8. Baydur A, Cha EJ, Sassoon CS. Validation of esophageal balloon technique at different lung volumes and postures. J Appl Physiol 1987;62(1):315-321.

9. Kárason S, Karlsen KL, Lundin S, Stenqvist O. A simplified method for separate measurements of lung and chest wall mechanics in ventilator-treated patients. Acta Anaesthesiol Scand 1999;43(3):308-315.

10. Plataki M, Hubmayr RD. Should mechanical ventilation be guided by esophageal pressure measurements? Curr Opin Crit Care 2011; 17(3):275-280.
11. McGinn T, Wyer PC, Newman TB, Keitz S, Leipzig R, For GG. Tips for learners of evidence-based medicine. 3. Measures of observer variability (kappa statistic). CMAJ 2004;171(11):1369-1373.

12. Pelosi P, Goldner M, McKibben A, Adams A, Eccher G, Caironi P, et al. Recruitment and derecruitment during acute respiratory failure: an experimental study. Am J Respir Crit Care Med 2001;164(1):122130

13. Washko GR, O'Donnell CR, Loring SH. Volume-related and volume-independent effects of posture on esophageal and transpulmonary pressures in healthy subjects. J Appl Physiol 2006;100(3):753758.

14. O'Brien RM. A caution regarding rules of thumb for variance inflation factors. Qual Quan 2007;41:673-690.

15. Pelosi P, Luecke T, Rocco PR. Chest wall mechanics and abdominal pressure during general anaesthesia in normal and obese individuals and in acute lung injury. Curr Opin Crit Care 2011; 17(1):72-79.

16. The Acute Respiratory Distress Syndrome Network. Ventilation with lower tidal volumes as compared with traditional tidal volumes for acute lung injury and the acute respiratory distress syndrome. N Engl J Med 2000;342(18):1301-1308.

17. Walter SD. Statistical significance and fragility criteria for assessing a difference of two proportions. J Clin Epidemiol 1991;44(12):13731378. I. Definitions. Intensive Care Med 2006;32(11):1722-1732.

This article is approved for Continuing Respiratory Care Education credit. For information and to obtain your CRCE

(free to AARC members) visit

\section{www.rcjournal.com}

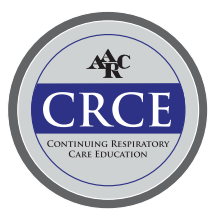

